\title{
Bio-inspired ant colony optimization based clustering algorithm with mobile sinks for applications in consumer home automation networks
}

Article

Accepted Version

Wang, J., Cao, J., Li, B., Lee, S. and Sherratt, S. (2015) Bioinspired ant colony optimization based clustering algorithm with mobile sinks for applications in consumer home automation networks. IEEE Transactions on Consumer Electronics, 61 (4). pp. 438-444. ISSN 0098-3063 doi: https://doi.org/10.1109/TCE.2015.7389797 Available at https://centaur.reading.ac.uk/54673/

It is advisable to refer to the publisher's version if you intend to cite from the work. See Guidance on citing.

Published version at: http://ieeexplore.ieee.org/xpl/articleDetails.jsp?arnumber $=7389797$

To link to this article DOI: http://dx.doi.org/10.1109/TCE.2015.7389797

Publisher: IEEE

All outputs in CentAUR are protected by Intellectual Property Rights law, including copyright law. Copyright and IPR is retained by the creators or other copyright holders. Terms and conditions for use of this material are defined in the End User Agreement. 


\section{www.reading.ac.uk/centaur}

\section{CentAUR}

Central Archive at the University of Reading

Reading's research outputs online 


\section{Full-Text version}

Title: Bio-inspired Ant Colony Optimization based Clustering Algorithm with Mobile Sinks for Applications in Consumer Home Automation Networks

Authors: Jin Wang, Member, IEEE

College of Information Engineering, Yangzhou University, Yangzhou 225009, China

(e-mail: jinwang@yzu.edu.cn)

Jiayi Cao,

College of Information Engineering, Yangzhou University, Yangzhou 225009, China

(e-mail: jycao@yzu.edu.cn)

Bin $\mathrm{Li}$,

College of Information Engineering, Yangzhou University, Yangzhou 225009, China

(e-mail: $\underline{\text { lb@yzu.edu.cn) }}$

Sungyoung Lee, Member, IEEE

Computer Engineering Department, Kyung Hee University, Suwon 449-701, Korea

(e-mail: sylee@khu.ac.kr)

R. Simon Sherratt, Fellow, IEEE

School of Systems Engineering, the University of Reading, RG6 6AY, UK

(e-mail: sherratt@ieee.org)

Publication: IEEE Transactions on Consumer Electronics

Volume: $\quad 61$

Issue: $\quad 4$

pp.: $\quad 438-444$

Date: $\quad$ November 2015

DOI: $\quad \underline{10.1109 / T C E .2015 .7389797}$

This work was supported by the National Natural Science Foundation of China (61402234, 61472344, 61402235, 61173072), the Natural Science Foundation of Jiangsu Province (BK2012461), the Project of six personnel in Jiangsu Province (2013-WLW-012, 2011-DZXX-032). It was also supported by the Industrial Strategic Technology Development Program (10041740) by the Ministry of Trade, Industry and Energy (MOTIE) Korea and the Industrial Core Technology Development Program (10049079) funded by the MOTIE Korea 


\begin{abstract}
With the fast development of wireless communications, ZigBee and semiconductor devices, home automation networks have recently become very popular. Since typical consumer products deployed in home automation networks are often powered by tiny and limited batteries, one of the most challenging research issues is concerning energy reduction and the balancing of energy consumption across the network in order to prolong the home network lifetime for consumer devices. The introduction of clustering and sink mobility techniques into home automation networks have been shown to be an efficient way to improve the network performance and have received significant research attention.

Taking inspiration from nature, this paper proposes an Ant Colony Optimization (ACO) based clustering algorithm specifically with mobile sink support for home automation networks. In this work, the network is divided into several clusters and cluster heads are selected within each cluster. Then, a mobile sink communicates with each cluster head to collect data directly through short range communications. The ACO algorithm has been utilized in this work in order to find the optimal mobility trajectory for the mobile sink. Extensive simulation results from this research show that the proposed algorithm significantly improves home network performance when using mobile sinks in terms of energy consumption and network lifetime as compared to other routing algorithms currently deployed for home automation networks.
\end{abstract}

\title{
Index Terms
}

Ant Colony Optimization, Clustering, Home Automation Networks, Sink Mobility.

\section{INTRODUCTION}

With the rapid development of low-cost and low-power devices and communication technologies, many kinds of home consumer products have been deployed to create home automation networks [1]. The home consumer products are usually distributed in the home environment to collect data, e.g. temperature and humidity, and then send their sensed data to a base station via wireless communication.

ZigBee has been shown to be suitable for implementing home automation networks because a mesh of sensors can be deployed to collect home data in a distributed, intelligent and collaborative manner [2]. Wireless Sensor Networks (WSNs) for Home Automation are primarily based on the ZigBee technology due to their intrinsic self-organizing and collaborative nature [3]. Tiny low-cost sensors can collect raw data from their surrounding areas and then transmit them to a remote sink node or base station in a distributed and collaborative way.

Since many home network sensors are typically powered by tiny irreplaceable batteries which usually cannot be recharged, the design of an energy efficient routing algorithm is an important research issue for home automation networks [4].

The clustering technique has been shown to be an effective way to reduce and balance energy consumption [5]-[8]. In clustering based algorithms, the whole network is divided into several clusters and a Cluster Head $(\mathrm{CH})$ is chosen to act as a local coordinator for each cluster. However, a hot spot problem will arise because some sensors close to the sink will have the burden of more traffic, thus depleting their energy quicker than other sensors far away from sink [5]. 
The introduction of sink mobility into WSNs has been shown to be a very efficient way to mitigate the hot spot problem and balance energy consumption [9]-[13]. To achieve sink mobility, sink nodes physically move throughout the network. The mobility may be achieved when the sink is attached to vehicles, animals or even people so that they can roam around the sensing field to collect data from sensors using very short range communications. Significant energy savings have been achieved offering long network lifetime [14]-[15].

Ant Colony Optimization (ACO) [16]-[18] is a bio-inspired algorithmic technique inspired by the behavior of ant colonies and this work postulates and demonstrates ACO is applicable to the guidance of sink mobility. The ants only have very limited local capability, but they can achieve globally optimal performance, e.g. finding the shortest path from their nest to a food source. Ants release pheromones to mark their path as they traverse their journey. The probability of an ant choosing a specific path is proportional to the concentration of pheromone which itself varies via evaporation or reinforcement. Through this mechanism, ants can finally find an optimal path from their nest to a food source. The first ant optimization systems were applied to combinatorial optimization problems, such as the famous Travelling Salesman Problem (TSP) and also quadratic assignment problems.

In this paper, a novel routing algorithm is proposed which combines ACO, clustering and sink mobility techniques in order to extend the lifetime of consumer home automation networks and improve their performance. The whole network is divided into several clusters and a $\mathrm{CH}$ is chosen in each cluster. Then, a mobile sink will communicate with all $\mathrm{CHs}$ and collect data from them through short range communications. The ACO algorithm is used to find the optimal sink mobility trajectory to improve network performance.

The rest of this paper is organized as follows. Section II discusses the relevant literature. Section III presents the system model for this research. In Section IV, the conventional ACO algorithm is briefly described, and Section $\mathrm{V}$ provides the proposed routing algorithm. Extensive simulation results are illustrated and compared in Section VI and Section VII concludes the paper.

\section{RELATED WORKS}

\section{A. Clustering Algorithms}

Low Energy Adaptive Clustering Hierarchy (LEACH) [5] is a famous hierarchical routing algorithm for WSNs which has become a benchmark for performance measurements in WSNs. In LEACH, sensor nodes are organized to form clusters with one $\mathrm{CH}$ in each cluster. $\mathrm{CHs}$ collect data from their member nodes, perform data processing, aggregation and then forward the data to a Base Station (BS) or sink node. To balance the energy dissipation among sensors, the role of the $\mathrm{CH}$ is rotated amongst sensors. LEACH can prolong the network lifetime in the order of 8-fold compared to traditional routing protocols. However, $\mathrm{LEACH}$ requires all $\mathrm{CHs}$ to perform direct transmissions to the $\mathrm{BS}$, which is not applicable to networks deployed in large regions.

Hybrid Energy-Efficient Distributed (HEED) [6] is an improved version of LEACH where a CH is selected via a primary parameter (e.g. residual energy), and a secondary parameter (e.g. intra-cluster communication cost.) Multi-hop inter-cluster communication is adopted. HEED terminates after a given number of iterations, and it can achieve a fairly uniform $\mathrm{CH}$ distribution inside the network. However, a hot spot problem emerges since nodes close to the sink need to relay more traffic through them compared to nodes far from the sink. 
A distributed and energy-aware clustering algorithm named Energy-efficient Clustering (EC) has been proposed [7]. In EC, sensor nodes are organized to form clusters with unequal size based on the hop distance to data sink. EC can effectively control cluster sizes by adapting the probability that a node becomes a $\mathrm{CH}$. Finally, traffic load and energy consumption are balanced and network lifetime is prolonged.

Unequal Cluster-based Routing (UCR) algorithm has been proposed [8]. Sensor nodes are grouped into clusters of unequal sizes. The size of the cluster increases as the distance of its $\mathrm{CH}$ from the base station increases. Therefore, clusters far away from the base station can preserve energy for the purpose of inter-cluster data forwarding. UCR also implements an energy-efficient multi-hop routing protocol for inter-cluster communication, which considers the trade-off between energy cost and sensor residual energy.

\section{B. Sink Movement Strategies}

The idea of using a mobile sink for WSNs was first proposed by Shah et al. [9], where the sink nodes were originally called "data MULEs". The MULEs traversed the sensing field randomly to collect data from nearby sensors. Then, MULEs locally buffered the collected data to then subsequently deliver the data to a wired access point in due course. This scheme was novel and easy to be implemented. The energy consumption of sensor nodes can be significantly reduced because the transmission range between the node and the MULE is significantly reduced.

Ma, Yang and Zhao [10] proposed a new data-gathering mechanism for large-scale WSNs by exploiting sink mobility. The mobile sink, termed M-collector, started the data-gathering tour periodically starting from a static data sink. It traversed the sensing field to collect data from the nearby nodes, and then uploaded their collected data to the sink when it returned to the starting point. For applications with strict distance and/or time constrains, the authors also proposed their improved data-gathering algorithm which utilized multiple M-collectors. Each of the M-collectors traversed through a shorter sub-tour rather than the entire tour.

A novel mobility scheme termed Set Packing Algorithm and TSP (SPAT) was proposed [11] to enable reliable and efficient data gathering for typical wireless sensor and actuator networks (WSANs). SPAT was based on the set packing algorithm and the common TSP to access all sensor nodes in the target network efficiently. By using SPAT, users were able to control all sensor hosts in an effective and real-time manner.

Konstantopoulos, Pantziou and Gavalas [12] proposed the MobiCluster protocol to minimize overall network overhead and energy consumption. In this protocol, cluster structures are built and $\mathrm{CHs}$ collect data from sensor nodes and perform a data filtering action on the collected raw data. Then, $\mathrm{CHs}$ transmitted their data to their assigned Rendezvous Nodes (RNs) which typically were located in proximity to the trajectory of the mobile sinks. The mobile sinks traversed the network and collected data from RNs when they were in a nearby area.

A unified framework was proposed by $\mathrm{Gu}, \mathrm{Ji}$ and $\mathrm{Li} \mathrm{[13]} \mathrm{to} \mathrm{analyze} \mathrm{the} \mathrm{sink} \mathrm{mobility} \mathrm{problem} \mathrm{with}$ delay constraints for WSNs. Other problems such as zero delay, infinite speed and connected sink sites were also discussed and relevant optimal algorithms were developed. The authors generalized their solutions and proposed a polynomial-time optimal algorithm to solve the problems for WSNs.

A novel joint optimization algorithm, combining clustering and sink node deployment for smart home networks was proposed by Wang et al. [14]. The authors' proposed two sink mobility based energy 
efficient clustering algorithms termed EMCA and MECA to reduce the average energy consumption and prolong network lifetime. Parameters including mobile sink number, velocity and moving trajectory were carefully studied with extensive simulation results and comparison to show an improvement to LEACH.

Chanak et al. [15] proposed a mobile sink based obstacle avoidance routing scheme for home automation networks. Obstacles were detected by sensors using IR communication and the sink moved to improve the data transmission to the sink node. The mobile sink also avoids obstacles to find the shortest path to collect data from static sensor nodes. The performance was well validated via ZigBee enabled hardware and a prototype test-bed.

\section{Ant Colony Optimization based Protocols}

ACO belongs to the class of biologically inspired heuristics. The basic idea of ACO-based algorithms is based on how ants search for food from their nest and finally find the optimal solution such as the shortest path from the nest to a food source.

Lee and Lee [16] proposed an Ant-Colony-Based Schedule Algorithm (ACB-SA) to solve the Efficient-Energy Coverage (EEC) problem. A new initialization method for pheromone field and the modified construction graph was adopted to improve the performance of this algorithm. In order to solve the EEC problem through a more realistic approach, this algorithm utilized a probability sensor detection model and applied ACB-SA to a heterogeneous sensor set.

With the aim to maximize the lifetime of heterogeneous WSNs, Lin and Zhang [17] proposed an ACO-based approach for Maximizing the Number of Connected Covers, named ACO-MNCC. By always pursing one more connected cover than the best-so-far solution, this approach searches for the optimal solution. The pheromone and the heuristic information were also designed to accelerate the search process. In the meantime, a local search procedure was developed to further improve the searching efficiency.

Gan, Guo and Chang [18] proposed an ACO algorithm based on the scout characteristic to solve the stagnation behavior and premature convergence problem of the common TSP. In this algorithm, artificial ants were partitioned into two groups: scout ants and common ants. By using a path evaluation model, the scout ants calculated each route's mutation probability of current optimal solution and searched around the optimal solution according to the mutation probability.

\section{SYSTEM MODEL}

The Home Automation network model used for this research is shown in Fig. 1. As can be seen in Fig. 1 , various types of sensor nodes (cameras, bio-sensors, RFID and mobile sink nodes) are deployed in a home network environment. It is assumed that cameras can only be installed in public areas including the dining room and sitting room for privacy reasons. Bio-sensors can be attached on or around people to collect human physical and physiological information. Sink nodes can be attached on users or implemented as mobile robots/vehicles to collect data from various sensors. A home gateway server is implemented which can communicate directly with both inside and outside home devices via wired or wireless connectivity. 


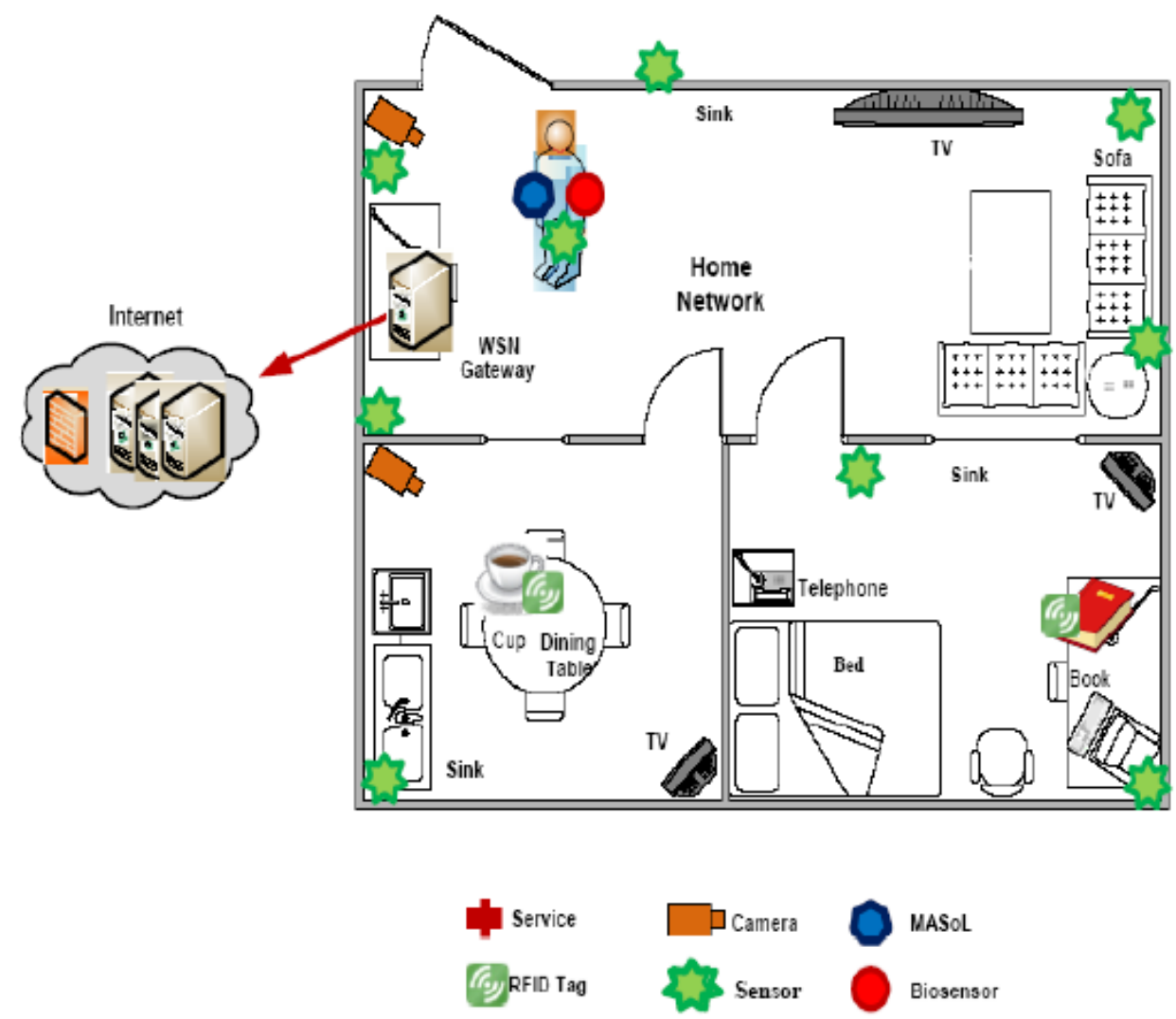

Fig. 1. Home automation network model.

The home network can be modeled as a graph $G=\langle V, E\rangle$ where $V$ is the set of sensors and $E$ is the set of links $(i, j)$. Here, $i$ and $j$ denote the neighboring nodes. Nodes $i$ and $j$ can communicate with each other directly if the Euclidean distance between them is smaller than the reliable reception radius. There is no obstacle between nodes.

A first order radio model [5] has been adopted in this research. If the reliable reception distance is larger than the threshold $d_{0}$, the multi-path ( $d^{4}$ power loss) model is used; otherwise, the free-space ( $d^{2}$ power loss) model is used. Therefore, the energy spent to transmit an $l$-bit message over distance $d$ can be calculated as:

$$
E_{T x}(l, d)= \begin{cases}l E_{\text {elec }}+l \varepsilon_{f s} d^{2} & d<d_{0} \\ l E_{\text {elec }}+l \varepsilon_{m p} d^{4} & d \geq d_{0}\end{cases}
$$

where $E_{\text {elec }}$ denotes the energy dissipated in the electronic circuitry, $\varepsilon_{f s}$ and $\varepsilon_{m p}$ denote the amplifier energy for free-space and multi-path conditions, and $d_{0}=\sqrt{\varepsilon_{f s} / \varepsilon_{m p}}$ when the above two formulae are equal. To receive an $l$-bit message, the following amount of energy will be consumed:

$$
E_{R x}(l)=l \cdot E_{\text {elec }}
$$


The following assumptions regarding the system model are made in this paper:

- Sensor nodes are homogeneous;

- Sensor nodes have the same initial energy;

- Wireless links are bi-directional and symmetric;

- The sink node is energy unconstrained and has free movement;

- There is no obstacle between each pair of sensor nodes.

\section{Conventional ACO Algorithm}

ACO algorithm can be applied to solve many combinatorial optimization problems, such as the TSP and quadratic assignment problems which aim to find globally optimal solution through interaction of local collaboration from ants and salesman etc.

In the ACO algorithm, it is assumed that there are $\mathrm{N}$ destinations and $\mathrm{M}$ ants. The probability of each ant to choose the next destination is proportional to the intensity of pheromone and is inversely proportional to the distance between the current destination and the next destination. At time $t$, the transmission probability from destination $i$ to destination $j$ for the $k^{\prime}$ th ant is calculated as:

$$
p_{i j}^{k}(t)= \begin{cases}\frac{\left[\tau_{i j}(t)\right]^{\alpha}\left[\eta_{i j}\right]^{\beta}}{\sum_{k \in \text { allowed }_{k}}\left[\tau_{i k}(t)\right]^{\alpha}\left[\eta_{i k}\right]^{\beta}} & \text { if } j \in \text { allowed }_{k} \\ 0 & \text { otherwise }\end{cases}
$$

where allowed denotes the set of destinations that have not been visited, $\tau_{i j}$ represents the intensity of path pheromone between destination $i$ and destination $j . \eta_{i j}$ is the heuristic desirability which is calculated as $\eta_{i j}=1 / d_{i j}$, where $d_{i j}$ denotes Euclidean distance. $\alpha$ and $\beta$ are constant parameters which refer to the influence of the pheromone and heuristic on the decision taken by the ant. After time $h, \tau_{i j}$ is updated as:

$$
\tau_{i j}(t+h)=(1-\rho) * \tau_{i j}(t)+\Delta \tau_{i j}
$$

where $\rho$ is the pheromone evaporation rate and the pheromone field is updated after all the ants finish their tours. $\Delta \tau_{i j}$ is the sum of pheromones emitted by all the ants and is:

$$
\Delta \tau_{i j}=\sum_{k=1}^{M} \Delta \tau_{i j}^{k}
$$

where, $\Delta \tau_{i j}^{k}$ is the quantity of the pheromone per unit length left by ant $k$ on the path and can be expressed as:

$$
\Delta \tau_{i j}^{k}= \begin{cases}Q / d_{i j} & \text { if } k \text {-h ant has used edge }(i, j) \\ 0 & \text { otherwise }\end{cases}
$$

After a given iteration number, this process will terminate and the optimal path can be found. 


\section{The Proposed Algorithm}

In this section, a mobile sink is utilized to collect data from sensor nodes. Clustering and ACO algorithms are combined together to improve the home network performance.

\section{A. Cluster Formation}

During this phase, the whole network is partitioned into several clusters and only one sensor node in each cluster serves as a $\mathrm{CH}$. To achieve this, a clustering algorithm which is a variation of LEACH is adopted. In this algorithm, sensor nodes with more residual energy will have higher probability to become CHs. In the meantime, with regards to the case that the distance between two candidate CHs is less than their reliable reception range, then the candidate $\mathrm{CH}$ with the most residual energy becomes the actual selected $\mathrm{CH}$ and the other candidate $\mathrm{CH}$ is relegated to an ordinary node. The detail of the process is as follows:

At the beginning of each round, each sensor node generates a random number between 0 and 1 , and then compares it with a predefined threshold $T(n)$. If the number is less than $T(n)$, and there are no other $\mathrm{CHs}$ in its transmission range, then this sensor node selects itself as a $\mathrm{CH}$ for the current round. However, when there is another $\mathrm{CH}$ in its transmission range, the one with more residual energy becomes final $\mathrm{CH}$ and the other one becomes a member node. The predefined threshold $T(n)$ is given as:

$$
T(n)= \begin{cases}\frac{p}{1-p^{*}(r \bmod 1 / p)} \cdot \frac{E_{\text {current }}}{E_{\text {initial }}} & \text { if } n \in G \\ 0 & \text { otherwise }\end{cases}
$$

where $P$ is the desired percentage of cluster heads, $r$ is the round number and $G$ is the set of nodes that have not been selected as cluster heads in the last $1 / p$ rounds. $E_{\text {current }}$ denotes the residual energy of nodes and $E_{\text {initial }}$ denotes the initial energy of nodes.

After selecting itself as a $\mathrm{CH}$, the node broadcasts a $\mathrm{CH}$ message to other ordinary sensor nodes. Those ordinary sensor nodes will join the $\mathrm{CH}$ according to the signal strength. After the clusters are formed, the $\mathrm{CHs}$ will create and broadcast a time division multiple-access schedule to exchange data with member nodes without collision.

\section{B. ACO based Sink Moving Strategy}

In order to mitigate the hot spot problem and extend the lifetime of the consumer home network, a mobile sink is utilized to traverse the network and collect data directly from CHs. At the same time, the network latency is thus decreased. In order to achieve this objective, the ACO algorithm is utilized to determine the mobility strategy. In this paper, the mobile sink is treated as a salesman and the CHs as a destination that needs to be visited. Therefore, this problem can be mapped to the TSP, which can be solved effectively by the ACO algorithm. The mobile sink will traverse the optimal path determined by the ACO algorithm. The mobile sink specifically visits the positions of $\mathrm{CHs}$ to collect data from $\mathrm{CHs}$ using single hop physically close communications. Since the transmission range is relatively short between the sink and nearby $\mathrm{CH}$, energy consumption of cluster heads can thus be largely decreased. 
The flowchart of the proposed algorithm is shown in Fig. 2. Some CHs will first be chosen based on improved LEACH, where $\mathrm{CHs}$ collect raw data from sensors in each cluster. Then, ACO algorithm is run to find the optimal path for mobile sink to visit the CHs. In this way, improved network performance can be achieved.

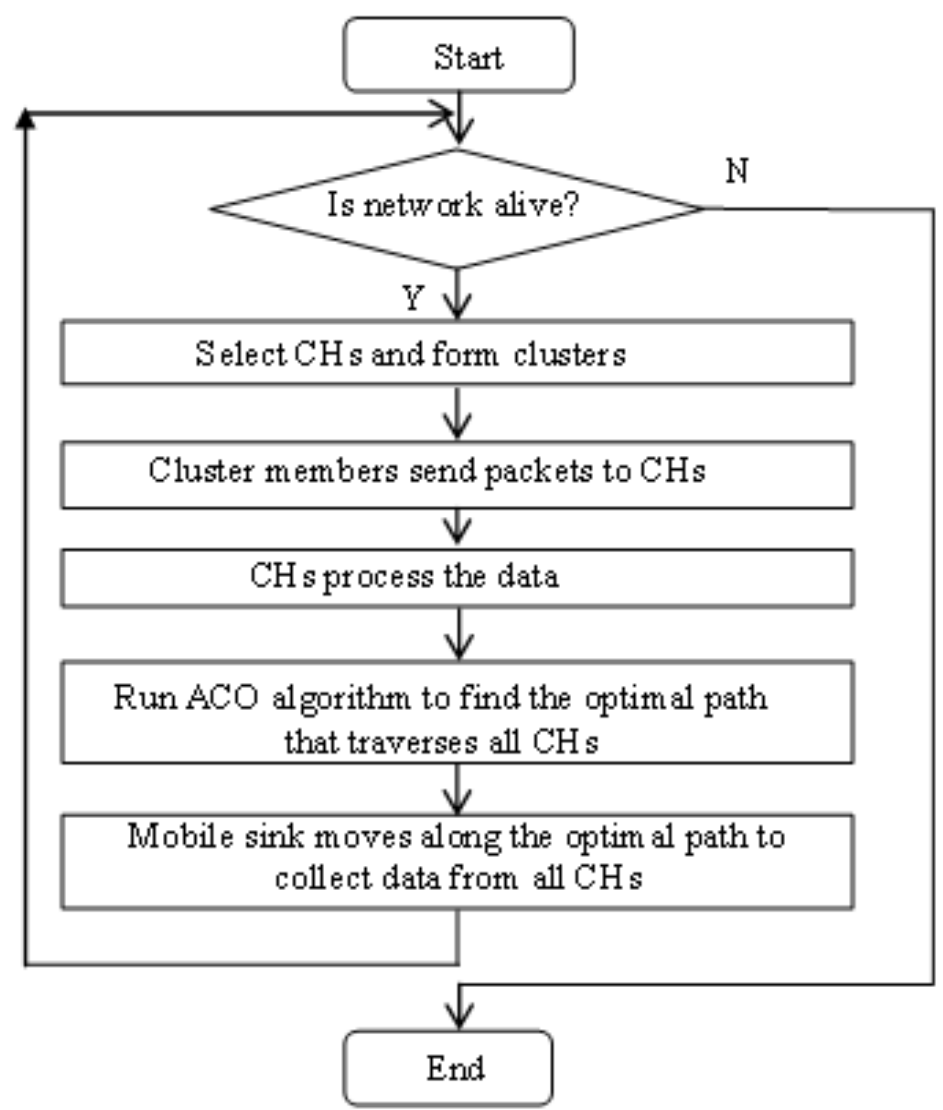

Fig. 2. Flowchart of the clustering algorithm presented in this work.

\section{Vi. Performance Evaluation}

To evaluate home automation network performance, the proposed algorithm is compared with two common algorithms from the literature, namely the LEACH and Mobile-P algorithms.

In the experiments, the whole network consists of a number of sensors between $[50,200]$ in a $100 \times 100 \mathrm{~m}^{2}$ area. All sensor nodes have the same initial energy of $0.5 \mathrm{~J}$ and they are randomly deployed inside the network. Relevant network parameters are listed in TABLE I.

Extensive simulations have been performed to determine suitable values for $\alpha$ and $\beta$, as listed in TABLE II. From TABLE II, it can be seen that when $\alpha, \beta$ and $\rho$ have values of 1,3 and 0.5 , the length of path is the shortest. Therefore, these three parameters are chosen as above in this paper. 
TABLE I

NETWORK PARAMETERS

\begin{tabular}{|c|c|}
\hline Parameter name & Value \\
\hline Network size & {$[100,100] \mathrm{m}^{2}$} \\
\hline Num. of nodes $(\mathrm{N})$ & {$[50,200]$} \\
\hline Transmission radius $\left(\mathrm{R}_{0}\right)$ & $50 \mathrm{~m}$ \\
\hline Packet length (1) & 2000 bits \\
\hline Initial energy $\left(\mathrm{E}_{0}\right)$ & $0.5 \mathrm{~J}$ \\
\hline $\begin{array}{l}\text { Energy consumption on } \\
\text { circuit }\left(\mathrm{E}_{\text {elec }}\right)\end{array}$ & $50 \mathrm{~nJ} / \mathrm{bit}$ \\
\hline $\begin{array}{l}\text { Free-space channel } \\
\text { parameter }\left(\varepsilon_{f s}\right)\end{array}$ & $10 \mathrm{pJ} / \mathrm{bit} / \mathrm{m}^{2}$ \\
\hline $\begin{array}{l}\text { Multi-path } \\
\text { parameter }\left(\varepsilon_{m p}\right)\end{array}$ & $0.0013 \mathrm{pJ} / \mathrm{bit} / \mathrm{m}^{4}$ \\
\hline Distance threshold $\left(d_{0}\right)$ & $\sqrt{\varepsilon_{f s} / \varepsilon_{m p}} \mathrm{~m}$ \\
\hline
\end{tabular}

TABLE II

LENGTH OF PATH BY USING DifFERENT VALUES FOR $\alpha, \beta$ AND $\rho$

\begin{tabular}{cccc}
\hline \hline$\alpha$ & $\beta$ & $\rho$ & $\begin{array}{c}\text { Length } \\
\text { of path }\end{array}$ \\
\hline 1 & 1 & 0.5 & 283.5245 \\
1 & 3 & 0.5 & 278.5245 \\
1 & 5 & 0.5 & 284.7612 \\
1 & 1 & 0.7 & 283.6792 \\
1 & 3 & 0.7 & 278.6730 \\
1 & 5 & 0.7 & 284.1543 \\
2 & 1 & 0.5 & 291.4423 \\
2 & 3 & 0.7 & 286.0665 \\
3 & 3 & 0.5 & 284.8403 \\
3 & 5 & 0.7 & 286.0928 \\
\hline
\end{tabular}

In Fig. 3, the overall energy consumption as a function of number of nodes is illustrated. From Fig. 3 , it can be seen that as the number of nodes increases then the energy consumption of all the algorithms increases accordingly. However, the algorithm proposed in this work has significant energy savings compared to the other two algorithms discussed, which means that the proposed algorithm is more suitable for the home network environment and especially as the consumer devices deployed in the home increases.

Lifetime is an important metric to evaluate home network performance. Comparison of the network lifetime in different conditions is shown in Fig. 4. It is clear that Mobile-P has a longer network lifetime than LEACH. However the algorithm proposed in this work has the longest network lifetime. This is because the mobile sink traverses the whole network and collects data from CHs directly through relatively short communication range. Thus, energy can be largely saved from network at the cost of more energy consumed by the moving sink node. Network lifetime can also be defined as the time when the first node dies, i.e. it runs out of residual energy. In Fig. 5, the lifetime of the network with different initial energy levels is illustrated for the above three algorithms. It might be expected that as the initial energy increases, the lifetime increases accordingly. However, as can be seen, the algorithm in this work lasts longer than the other algorithms tested. From Fig. 5, it can also be found that network lifetime of the proposed algorithm is approximately 1.5 times that of LEACH. 


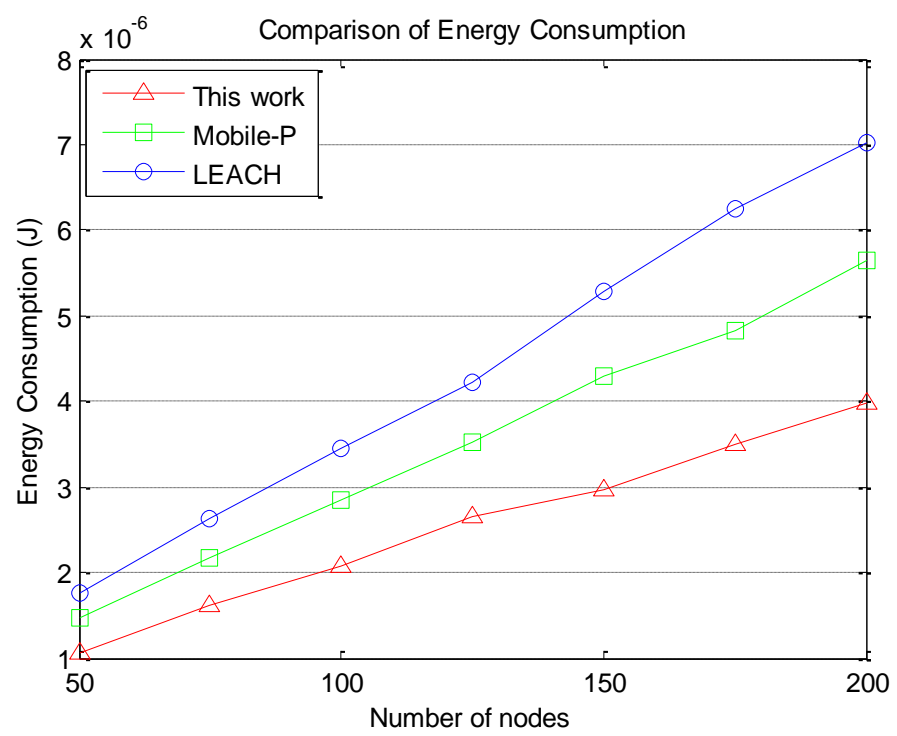

Fig. 3. Comparison of energy consumption.

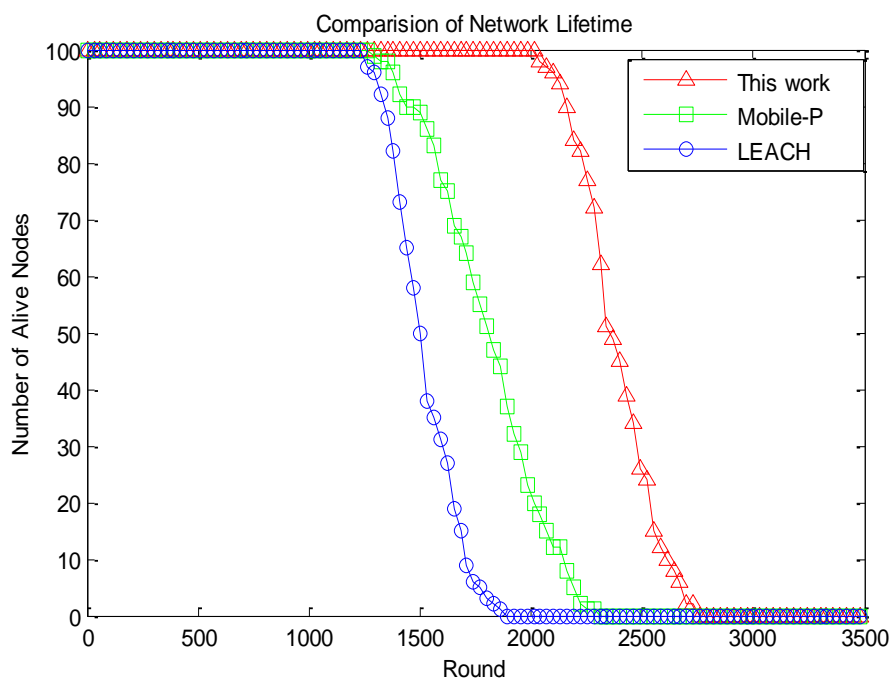

Fig. 4. Comparison of network lifetime.

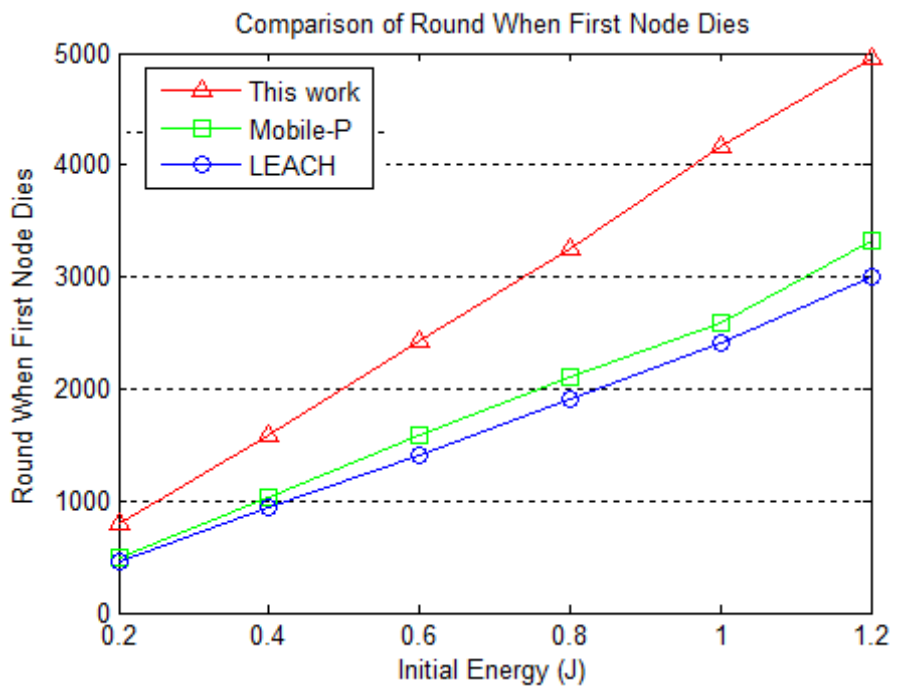

Fig. 5. Comparison of round when the first node dies. 
Fig. 6 depicts the comparison of residual energy for the three algorithms. In this proposed algorithm, $\mathrm{CHs}$ forward data to the mobile sink through a very short communication range, so the energy consumption is relatively low. In the other two algorithms, single-hop and multi-hop mechanisms need to be adopted when $\mathrm{CHs}$ transmit their data to the remote sink which consumes more energy than the proposed algorithm, especially in some large scale networks. Consequently, the residual energy of this proposed algorithm is larger than that of the other two algorithms.

The amount of packets received by the sink node is compared in Fig. 7. It is assumed that each sensor node generates an equal amount of data per time. Therefore, before the first node dies in LEACH, the amount of data packets sent to sink node is the same for three algorithms. After about 2100 rounds, networks using LEACH and Mobile-P algorithms are all out of function. However, the network with the proposed algorithm can still send data packets until about 2500 rounds. Compared with LEACH and using the same initial energy, this work receives 1.6 times more data packets sent though the network before the energy has expired.

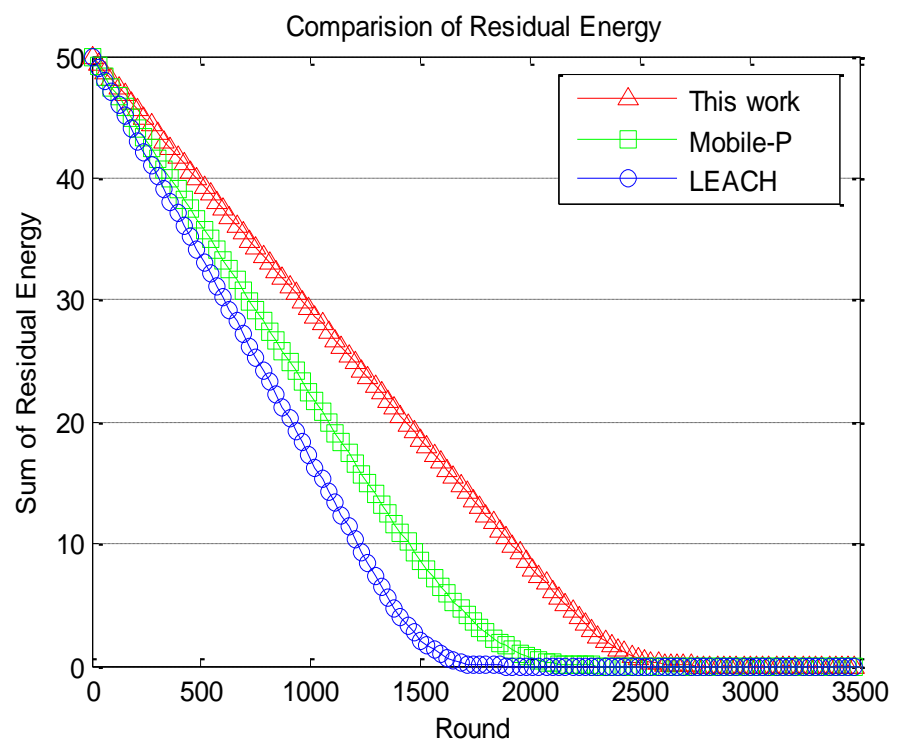

Fig. 6. Comparison of residual energy.

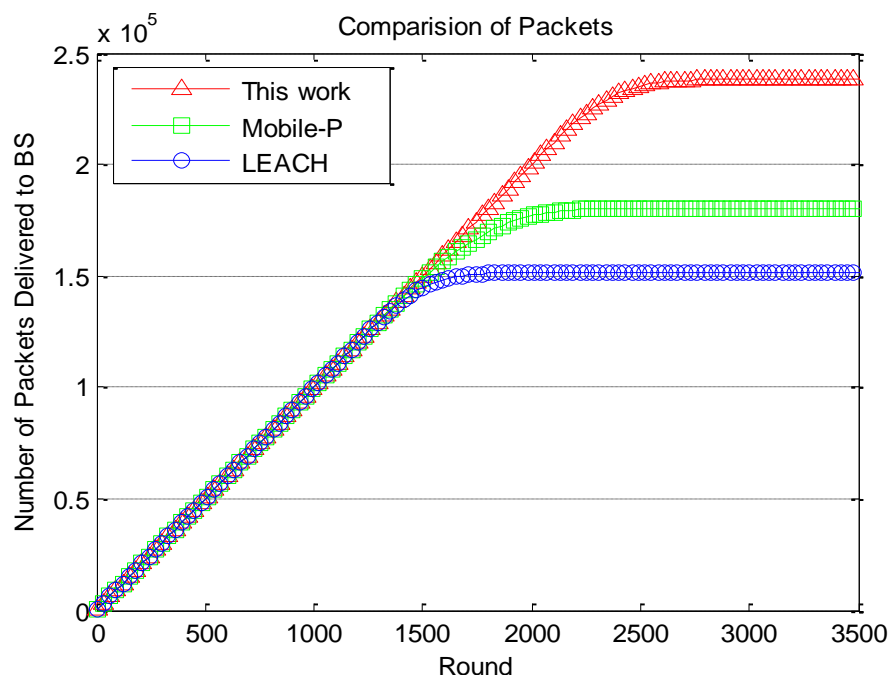

Fig. 7. Comparison of packet delivery. 


\section{CONCLUSIONS}

With the aim to prolong home automation network lifetime, a novel Ant Colony Optimization based clustering algorithm with Mobile Sink support is proposed in this paper. A clustering algorithm is used to partition network into several clusters and one cluster head is chosen inside each cluster. A mobile sink traverses the network collecting data via an optimal moving trajectory which is determined by ACO algorithm. Extensive simulation results also validate superiority of the proposed algorithm performance against the benchmark LEACH and Mobile-P algorithms.

This work has clear potential applications to reduce the energy and extend lifetime for battery operated devices in home automation networks. The concept of mobile sink can be extended to consumer devices attached on or around people, which is very suitable to applications including Home Healthcare and Ambient Assisted Living.

\section{REFERENCES}

[1] H. Oh, H. Bahn, and K. Chae, "An energy-efficient sensor routing scheme for home automation networks", IEEE Trans. Consum. Electron, vol. 51, no. 3, pp.836-839, Aug. 2005.

[2] J. Byun, B. Jeon, and J. Noh, "An intelligent self-adjusting sensor for smart home services based on ZigBee communications", IEEE Trans. Consum. Electron., vol. 58, no. 3, Aug. 2012.

[3] I. Akyildiz, W. Su, Y. Sankarasubramaniam, and E. Cayirci, "Wireless sensor networks: a survey", Journal of Computer Networks, vol. 38, no. 4, pp. 393-422, Mar. 2002.

[4] K. Akkaya and M. Younis, "A survey on routing protocols in wireless sensor networks", $A d$ Hoc Network Journal, Elsevier, vol. 3, no. 3, pp. 325-349, May 2005.

[5] W. R. Heinzelman, A. Chandrakasan, and H. Balakishnan, "Energy-efficient communication protocol for wireless microsensor networks", in Proc. 33rd Annual Hawaii Int. Conf. System Sciences, Dec. 2002.

[6] O. Younis and S. Fahmy, "HEED: a hybrid, energy-efficient, distributed clustering approach for ad hoc sensor networks", IEEE Trans. Mobile Comput., vol. 3, no. 4, pp. 366 -379, Oct. 2004.

[7] D. L. Wei, Y. C. Jin, and S. Vural, "An Energy-Efficient Clustering Solution for Wireless Sensor Networks", IEEE Trans. Wireless Commun., vol. 10, no. 11, pp. 3973-3983, Sept. 2011.

[8] G. Chen, C. Li, M. Ye, and J. Wu, "An unequal cluster-based routing protocol in wireless sensor networks", Wireless Networks, Kluwer, vol. 15, no. 2, pp. 193-207, Apr. 2009.

[9] R. C. Shah, S. Roy, S. Jain, and W. Brunette, "Data MULEs: modeling a three-tier architecture for sparse sensor networks", in Proc. ${ }^{\text {st }}$ IEEE Int. Workshop Sensor Network Protocols and Applications, Alaska, pp. 30-41, May. 2003.

[10] M. Ma, Y. Y. Yang, and M. Zhao, "Tour Planning for Mobile Data-Gathering Mechanisms in Wireless Sensor Networks", IEEE Trans. Veh. Technol., vol. 62, no. 4, pp. 1472-1483, Nov. 2013. 
[11] H. Nakayama and Z. M. Fadlullah, and N. Ansari, "A Novel Scheme for WSAN Sink Mobility Based on Clustering and Set Packing Techniques", IEEE Trans. Autom. Control, vol. 56, no. 10, pp. 2381-2389, Aug. 2011.

[12] C. Konstantopoulos, G. Pantziou, and D. Gavalas, "A Rendezvous-Based Approach Enabling Energy-Efficient Sensory Data Collection with Mobile Sinks", IEEE Trans. Parallel Distrib. Syst., vol. 23, no. 5, pp. 809-817, Sept. 2012.

[13] Y. Gu, Y. S. Ji, and J. Li, "ESWC: Efficient Scheduling for the Mobile Sink in Wireless Sensor Networks with Delay Constraint", IEEE Trans. Parallel Distrib. Syst., vol. 24, no. 7, pp. 13101320, Jul. 2013.

[14] J. Wang, Y. Yin, J. Zhang, S. Lee, and R. S. Sherratt, "Mobility based energy efficient and multi-sink algorithm for consumer home networks", IEEE Trans. Consum. Electron., vol. 59, no. 1, pp.77-84, Feb. 2013.

[15] P. Chanak, I. Banerjee, J. Wang, and R. S. Sherratt, "Obstacle Avoidance Routing Scheme Through Optimal Sink Movement for Home Monitoring and Mobile Robotic Consumer Devices", IEEE Trans. Consum. Electron., vol. 60, no. 4, pp. 596 - 604, Nov. 2014.

[16] J. W. Lee and J. J. Lee, "Ant-Colony-Based Scheduling Algorithm for Energy-Efficient Coverage of WSN", IEEE Sensors J., vol. 12, no. 10, pp. 3036-3046, Jul. 2012.

[17] Y. Lin and J. Zhang, "An Ant Colony Optimization Approach for Maximizing the Lifetime of Heterogeneous Wireless Sensor Networks", IEEE Trans. Syst., Man, Cybern. C, Appl. Rev., vol. 42, no. 3, pp. 408-420, Apr. 2012.

[18] R. W. Gan, Q. S. Guo and H. Y. Chang, "Improved ant colony optimization algorithm for the traveling salesman problems", J. System Engineering and Electronics, vol. 21, no. 2, pp. 329333, Sept. 2010.

\section{BIOGRAPHIES}

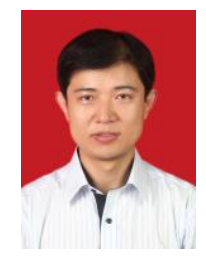

Jin Wang (M'11) received the B.S. and M.S. degree from Nanjing University of Posts and Telecommunications, China in 2002 and 2005, respectively. He received Ph.D. degree from Kyung Hee University Korea in 2010. Now, he is a professor in the College of Information Engineering, Yangzhou University. His research interests mainly include routing algorithm design, performance evaluation and optimization for wireless ad hoc and sensor networks. He is a Member of IEEE and ACM.

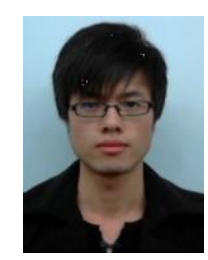

Jiayi Cao received the bachelor degree in computer science and technology major from the Guangling College of Yangzhou University in 2014. He is currently pursuing master degree in the College of Information Engineering, Yangzhou University. His research interests include energy efficient routing algorithms for wireless sensor networks.

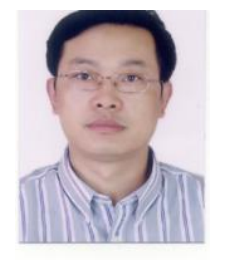

Bin Li received the B.S. degree in Computer Software from Fudan University, China in 1986, M.S. and Ph.D. degrees in Computer Application Technology from Najing University of Aeronautics \& Astronautics, China in 1993 and 2001 respectively. He is now a professor in Yangzhou University. He has published more than 100 journal and conference papers. His main research interests include artificial intelligence, multiagent system and service oriented computing. 
Sungyoung Lee (M'05) received his B.S. from Korea University, Seoul, Korea. He got his M.S. and Ph.D. degrees in Computer Science from Illinois Institute of Technology (IIT), Chicago, USA in 1987 and 1991 respectively. He has been a professor in the department of Computer Engineering, Kyung Hee University, Korea since 1993. His current research focuses on Ubiquitous Computing and Applications, Wireless Ad-hoc and Sensor Networks, Context-aware Middleware etc. $\mathrm{He}$ is a Member of IEEE and ACM.

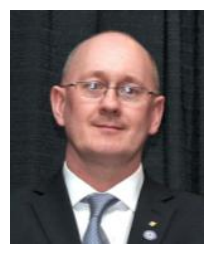

R. Simon Sherratt (M'97-SM'02-F'12) received the B.Eng. degree in Electronic Systems and Control Engineering from Sheffield City Polytechnic, UK in 1992, M.Sc. in Data Telecommunications in 1994 and Ph.D. in video signal processing in 1996 from the University of Salford, UK.

In 1996, he was appointed as a Lecturer in Electronic Engineering at the University of Reading where he is now a Professor of Consumer Electronics and Head of Wireless and Computing research. His research topic is signal processing in consumer electronic devices.

Eur Ing Professor Sherratt was an IEEE Consumer Electronics Society Vice President (08-09) and a serving AdCom member (03-08, 10-15). He received the IEEE Chester Sall Memorial $1{ }^{\text {st }}$ Place Award in 2006 and is now the Editor-in-Chief of the IEEE TRANSACTIONS ON CONSUMER ELECTRONICS. 